\title{
¿Cómo percibe el alumnado universitario de educación física la evaluación recibida? Contraste de dos metodologías diferentes \\ How do the university physical education students perceive the assessment received? Contrast of two different methodologies

\author{
David Hortigüela Alcalá, Ángel Pérez Pueyo, Alejandro Salicetti Fonseca \\ Universidad de Burgos (España), Universidad de León (España), Universidad de Costa Rica (Costa Rica)
}

El objetivo de este estudio es analizar cuál es la percepción que tiene el alumnado sobre la evaluación recibida a lo largo del curso escolar, incidiendo en tres factores fundamentales: la responsabilidad individual y grupal, la regulación del trabajo durante el proceso y la autenticidad de los aprendizajes adquiridos vinculados a la vida real. Los participantes son 642 estudiantes de ocho asignaturas vinculadas al ámbito de la enseñanza de la Educación Física (EF) de dos Universidades españolas, de los cuales 307 han recibido una evaluación tradicional y 335 han participado en la estrategia evaluativa del reparto de notas. Se ha utilizado un cuestionario validado sobre metodología y evaluación en formación inicial, en el que se especifican diferentes ítems vinculados al tipo de evaluación recibida. Se ha empleado un análisis cuantitativo, dividiéndose en descriptivo: medias, desviación típica (DT) y varianzas para cada uno de los factores de análisis e inferencial: tablas de contingencia y $\bullet^{\circ}$, correlaciones y ANOVAS. Se ha observado cómo el alumnado que ha participado en el reparto de notas percibe el proceso de aprendizaje más guiado y coherente, asumiendo también que requiere de una responsabilidad mayor, tanto de uno mismo como para lo demás. Del mismo modo, se demuestra que el alumnado que más veces ha trabajado en grupo percibe el reparto de notas como una estrategia que facilita un aprendizaje más auténtico. Sin embargo, el curso en el que se encuentre el alumnado o su edad no determina su percepción respecto a esa autenticidad de los aprendizajes.

Palabras clave. Percepción del alumnado, reparto de notas, aprendizaje auténtico, evaluación tradicional, responsabilidad en el proceso.

\begin{abstract}
The aim of this study is to analyze the perception of the students on the evaluation received during the school year, focusing on three key factors: personal and group responsibility, labor regulation during the process and the authenticity of the acquired learning linked to real life. Participants were 642 students from eight school years related to Physical Education Teaching Education (PETE) of two Spanish Universities where 307 received a traditional evaluation and 335 participated in the assessment strategy of sharing notes. We used a validated questionnaire on evaluation methodology and initial training, in which there are different items related to the type of assessment received. The design followed a quantitative analysis, divided into descriptive: means, standard deviation and variances for each of the factors, and inferential analysis: contingency tables and $\div^{2}$, correlations and ANOVAS. Students who participated in the distribution of notes perceived the learning process more coherent and directed, assuming a greater personal responsibility for oneself and the others. Similarly, students who have worked in groups more times perceived the distribution of notes as a strategy that facilitates a more authentic learning. However, school year or age did not determine their perceptions regarding the authenticity of learning.
\end{abstract}

Key words. Perception of the students, sharing notes, authentic learning, traditional assessment, responsibility in the process.

\section{Introducción}

La Declaración de la Sorbona (1998), que dio comienzo a la andadura definitiva del Espacio Europeo de Educación Superior(EEES), incorporó el concepto de competencia al sistema educativo universitario.

Estas competencias vienen definidas como aquellas características de una persona que están relacionadas con el desempeño efectivo de un trabajo y que pueden ser comunes a otras muchas situaciones (Delamare Le Deist \& Winterton, 2005). Esta idea de competencias viene a otorgar al ámbito universitario el carácter de rigor necesario que determine un bagaje por parte del alumnado para demostrar determinadas destrezas y conocimientos.

El concepto de competencias, tanto transversales como específicas, se identifica como clave y variado aunque con aspectos comunes según diferentes autores (Argudín, 2000; Barnett, 2001; Bautista, Mora, \& Gata, 2005; Gil \& Chiva, 2014; González \& Wagenaar, 2003; Hernanz \& Rosselló, 2003; Heywood, Gonczi \& Hager, 1992; Perrenoud, 2004; Rivero \& Oliván, 2006; Rué \& Martínez, 2005; Salganik, Rychen, Moser \& Konstant, 1999; Zabalza, 2012). Entre esta homogeneidad de criterios se encuentra: a) necesidad de una constante tutorización entre profesor y alumnado, existiendo una retroalimentación constante, b) dotar de un carácter práctico a las tareas desdeun enfoquemultidisciplinar, c) búsqueda de la relación directa con actuaciones exitosas en la sociedad: cooperación, solución de problemas, procesamiento de la información...

Uno de los aspectos fundamentales que deriva del trabajo de las competencias en educación superior es la idoneidad de aprender a trabajar en grupo, tal y como establece en su análisis la ANECA a partir del proyecto Tuning. Este aspecto se encuentra relacionado con el término

Fecha recepción: 22-09-14- Fecha envío revisores: 25-09-14- Fecha de aceptación: 19-11-14 Ángel Pérez Pueyo angel.perez.pueyo@unileon.es que establece Delgado (2005) de competencias personales, definidas como esos conocimientos intrínsecos a la persona y extrapolables a la realidad social. Este tipo de habilidades de relación social y de integración en diferentes colectivos, permiten a las personas mejorar su interacción con los demás. Sin embargo, este tipo de actividad, que ha sido utilizada en el ámbito universitario desde mucho antes de la implantación del EEES, no suele ser, precisamente, la más valorada por el alumnado como actividad de calificación, determinándose en muchos casos como un proceso injusto (Trueba, 2010).

La evaluación formativa es considerada como una actividad sistemática y continua que tiene por objeto proporcionar la información necesaria sobre el proceso educativo. Sus fines son reajustar los objetivos, revisar críticamente los planes, los programas, los métodos y recursos, orientar a los estudiantes así como retroalimentar el proceso de enseñanza y aprendizaje (Boud \& Falchikoz, 2007; Harald \& Mulder, 2012; López-Pastor, 1999, 2008; Trigueros, Rivera \& De la torre, 2011). Por tanto, la evaluación formativa se entiende como un continuo que permite una mayor implicación del alumnado en las tareas que realice y un mayor criterio a la hora de evaluarse tanto a sí mismo como a los demás (Hammersley \& Orsmond, 2007; Sanmartí, 2007; Castro, Gómez \& Macazaga, 2014).

En este sentido, autores como Wimshurst and Manning (2012) destacan la importancia que tiene fomentar este tipo de evaluación de manera grupal, incidiendo en la propia evaluación del trabajo por cada uno de sus integrantes. Este planteamiento deberállevarse a cabo bajo la definición de unos criterios iniciales aceptados por todos los integrantes del grupo que les permitirá establecer las condiciones generales de trabajo imperantes durante todo el proceso (Loureiro, Pombo \& Moreria, 2012). Esta idea de trabajo difiere totalmente de modelos más tradicionales en los que se dedica un periodo de tiempo al final de cada asignatura para realizar pruebas y exámenes como único objetivo calificador (López-Pastor \& Gea, 2009; López-Pastor \& Palacios, 2012). 
En el trabajo en grupo pueden generarse diferentes problemáticas que afectan a su desarrollo armónico (Panitz, 1997; Tolmie et al., 2010), existiendo un comportamiento vinculado a la habilidad relacionada con las competencias transversales de carácter personal: el efecto polizón. Este comportamiento, definido por (Kerr \& Bruun 1983; Slavin; 1983) consiste según Velázquez (2013) en que algunos miembros del grupo, generalmente aquellos con menos habilidades en relación a la tarea que deben realizar, dejan que el resto de sus compañeros hagan la mayor parte del trabajo, beneficiándose de los logros grupales (p. 59). Por otro lado, Jiménez (2006) establece que este tipo de actuaciones desencadenan en situaciones en la que estudiantes académicamente superiores permiten que sus compañeros, con un nivel académico inferior, se aprovechen de sus esfuerzos. Este hecho es señalado por Velázquez (2013) como un problema asociado puesto que desde el comienzo existe una división disfuncional de la tarea (Sheingold, Hawkins \& Char, 1984) en la que los estudiantes más capaces asumen los roles de liderazgo, organizando y desarrollando todo el trabajo principal, mientras que el resto asume únicamente las tareas secundarias.

Una de las principales problemáticas que se crea en este tipo de trabajos es la renuncia o abandono de la tarea ante el más mínimo problema en su ejecución o con los compañeros, así como la gestión destructiva del conflicto. En este sentido Velázquez (2013) destaca que uno de los posibles motivos causantes puede ser la sensación de injusticia que se genera cuando se sabe que todos los integrantes del grupo van a obtener la misma nota a pesar de que no se han implicado ni trabajado de la misma manera.

Referido a la evaluación y no a la calificación, encontramos diferentes trabajos sobre la percepción que tiene el alumnado acerca de la participación en estos procesos. Algunas experiencias llevadas a cabo por varios autores (Agustí, Suárez-Ojeda \& Baeza, 2009, Chica, 2011; López-Pastor, Castejón \& Pérez-Pueyo, 2012; Badura, Millard \& Shah, 2008; Ponn, McNaught, Lam \& Kwan, 2009) han mostrado que tras poner en práctica un sistema de evaluación negociado y una coevaluación entre iguales, se produjo un incremento de la motivación hacia los contenidos abordados y un mayor implicación de la clase.

Cuando se trata de que el alumnado califique a sus compañeros, los estudios muestran que las perspectivas de valoración cambian, existiendo una mayor resistencia de la clase a la hora de poner una nota a sus iguales (Hamodi \& López, 2012; López-Pastor, 2008; McMahon, 2010). No obstante, esta percepción es diferente cuando existe un trabajo de grupo desde el comienzo en el que se determinan las tareas que tiene que realizar cada uno de los integrantes y se va llevando a cabo una coevaluación intragrupal durante el proceso, lo que provoca menor resistencia y mayor objetividad para evaluar a los compañeros (Liu \& Carless, 2006; Vernetta, López \& Delgado, 2009; Plaza, Bosque, Cerezo, Olmo \& Viseras 2012). Por tanto, a mayor implicación del alumnado en su propio trabajo a través de la adquisición de una responsabilidad, tanto individual como grupal, en las tareas que se propongan, mayor clima cooperativo en el aula (Jiménez, 2006; Trueba, 2010). Un estudio realizado por Carvalho (2012) reflejó que más del 70\% de los estudiantes se mostraron satisfechos con los procesos formativos de reparto de notas, asumiendo la discusión con los compañeros del grupo como algo habitual en determinadas fases del proceso.

Por todo ello, la integración de la evaluación formativa con los procesos de coevaluación intragrupal a través de los repartos de nota nos acercan al concepto de evaluación auténtica (Pérez-Pueyo, Julián \& López-Pastor, 2009). Las técnicas, instrumentos y situaciones de evaluación asociados a este concepto se relacionan de forma intensa con situaciones de aprendizaje reales o lo más vinculada posible al desarrollo profesional (Dochy, Segers \& Dierik, 2002; Maclellan, 2004; Torrance, 1994).

Así, en estudio, y a diferencia de otros que han utilizado este cuestionario, se contrasta la percepción del alumnado que ha recibido procesos metodológicos diferentes, centrados éstos en el sistema de evaluación y en el rol que desempeña el alumnado a lo largo del curso.

\section{Método}

\section{Objetivos}

-Conocer el grado de relación existente entre cada uno de los factores del cuestionario, relativos a la evaluación de aprendizajes auténticos, la claridad en el sistema de evaluación y la regulación del trabajo.

-Comprobar el grado de significatividad existente entre los aspectos vinculados a la responsabilidad individual y grupal y la regulación del trabajo que percibe el alumnado en cada uno de las dos vías de evaluación.

-Valorar en qué medida el número de veces que el alumnado haya participado en trabajos de grupo a lo largo de la carrera, la edad y el curso influyen en su percepción sobre el aprendizaje auténtico recibido en cada uno de los grupos.

\section{Participantes}

La muestra que compone el estudio es de 642 estudiantes (53.8\% varones y $46.2 \%$ mujeres). La media de edad es de 20.73 años (DT = 2.13). De la totalidad de los participantes 307 han recibido una evaluación tradicional y 335 han sido evaluados mediante le técnica del reparto de notas. Tras realizar la prueba de shapiro-wilk se observa que la muestra responde a la normalidad ( $p=.153$ ). Todos los estudiantes se reparten en ocho asignaturas de dos Universidades españolas. Las materias implicadas pertenecen a titulaciones relacionadas con la enseñanza de la EF, todas ellas distribuidas en el primer o el segundo semestre del curso escolar 2013-2014. Cada una de las asignaturas se divide en dos grupos (A y B), impartidos cada uno por un docente. En el grupo A se ha desarrollado una evaluación de carácter más tradicional, donde además de no existir una retroalimentación formativa a lo largo del proceso, el alumnado ha realizado trabajos y exámenes de manera individual. Es en el grupo $B$ en el que se ha desarrollado el reparto de notas, asociado éste a una evaluación formativa y trabajo grupal.

En la tabla 1 puede observarse la muestra utilizada en el estudio, dividida en casos, con su nombre, la Universidad a la que pertenece, curso y número de estudiantes en cada grupo

\section{Instrumentos}

El instrumento utilizado para la obtención de datos ha sido el cuestionario sobre metodología y evaluación en formación inicial, validado por Castejón, Santos y Palacios (2013), en concreto la Escala de Sistemas de Evaluación, que aborda diferentes enfoques relacionados con los sistemas de evaluación, calificación, percepción y participación del alumnado en procesos de evaluación formativa. Este cuestionario obtiene una fiabilidad según el alfa de cronbach de 84 .

El cuestionario final que se utilizó con los participantes tiene un total de 25 cuestiones, estructuradas bajo una escala Likert que se estructura en los rangos de respuesta de uno (Nada) hasta cinco (Mucho).

El análisis factorial realizado en la validación del cuestionario muestra tres factores:

1. Evaluación de aprendizajes auténticos (11 ítems). Consta de ítems vinculados con el aprendizaje adquirido por el alumnado en las asignaturas y su relación con la aplicación de esos conocimientos al ámbito diario y profesional.

2. Claridad en el sistema de evaluación (ocho ítems). En este factor se abordan cuestiones relativas a la delimitación de criterios de evaluación de las asignaturas y su comprensión y seguimiento por parte del alumnado.

3. Regulación del proceso y continuidad (seis ítems). Se abordan ítems relacionados con la asistencia del alumnado a lo largo de la asignatura y la necesidad de un seguimiento a lo largo de proceso de enseñanza y aprendizaje.

\section{Diseñoy procedimiento}

El estudio es de carácter expostfacto, ya que todos los participantes en cada uno de los grupos, una vez finalizadas las asignaturas, 
Tabla 1

\begin{tabular}{|c|c|c|c|c|c|}
\hline \multicolumn{6}{|c|}{ Grupo / tipo de evaluación / participantes } \\
\hline 1 & EF y su Didáctica & Burgos & $2^{\circ}$ & Grupo A/ Tradicional/47 & Grupo B/R. notas/51 \\
\hline 2 & Juego Educativo & Burgos & $4^{\circ}$ & Grupo A/ Tradicional/40 & Grupo B/R. notas/41 \\
\hline 3 & Desarrollo Psicomotor I & Burgos & $3^{\circ}$ & Grupo A/ Tradicional/38 & Grupo B/R. notas/42 \\
\hline 4 & Desarrollo Psicomotor II & Burgos & $4^{\circ}$ & Grupo A/ Tradicional/36 & Grupo B/R. notas/35 \\
\hline 5 & Psicomot & Bur & $4^{\circ}$ & $\mathrm{A} / \mathrm{Tr}$ & \\
\hline 6 & Expresión Corporal & Vallad. & $3^{\circ}$ & Grupo A/ Tra & Grupo B \\
\hline 7 & Es colar & Vall & $2^{\circ}$ & A $\operatorname{Tra}$ & Grupo B/R. notas/53 \\
\hline \multirow[t]{4}{*}{8} & Educación Física y Salud & Vallad. & $4^{\circ}$ & Grupo A/ Tradicional $/ 39$ & Grupo B/R. notas/37 \\
\hline & \multirow{2}{*}{\multicolumn{4}{|c|}{$\begin{array}{l}\text { Total de participantes en eval uación tradicional } \\
\text { Total de participan tes en reparto de notas }\end{array}$}} & \\
\hline & & & & & 35 \\
\hline & & \multicolumn{3}{|c|}{$\begin{array}{l}\text { TOTAL MUESTRA } \\
\text { TOTA }\end{array}$} & 642 \\
\hline
\end{tabular}

Tabla 2

Frecuencias y comparación de medias por factores para cada uno de los grupos (nivel de significación en \begin{aligned} las diferencias: $* p<.005) \\$\hline\end{aligned}

\begin{tabular}{ccccc}
\hline & $\mathrm{N}$ & \multicolumn{1}{c}{ Media } & Des. Tip & Vari \\
\hline \multicolumn{4}{c}{ Grupos evaluación tradicional } \\
\hline Factor 1 & 307 & 3.64 & .584 & .341 \\
Factor 2 & 307 & 4.14 & .267 & .071 \\
Factor 3 & 307 & 3.51 & .734 & .538 \\
\hline \multicolumn{4}{c}{ Grup os reparto de notas } \\
\hline Factor 1 & 335 & $4.63 *$ & .314 \\
Factor 2 & 335 & 4.19 & .324 & .098 \\
Factor 3 & 335 & $4.52^{*}$ & .351 & .104 \\
\hline
\end{tabular}

cumplimentaron el cuestionario. Éste fue respondido individualmente y de manera presencial en el aula. En todo momento se garantizó el anonimato para que las respuestas de los estudiantes fueran lo más sinceras posibles, así como la confidencialidad en el tratamiento de los datos.

A pesar de que cada asignatura se divide en dos grupos, uno impartido por cada profesor, los objetivos y competencias a adquirir en cada una son las mismas. Lo que difiere en cada uno de los grupos es el tipo de metodología empleado, analizándose en concreto el uso del reparto de notas. En este reparto de notas, el alumnado, dividido en grupos, al finalizar el proceso deberá consensuar la nota individual de cada integrante a partir de la total que el profesor les haya otorgado. Para ello, desde el comienzo se delimitan una serie de criterios de trabajo para cada uno de los grupos, que junto a la implantación de estrategias de autoevaluación y coevaluación intergrupales y el uso de instrumentos de seguimiento, permiten al alumnado desarrollar la autonomía y responsabilidad necesaria para reconocer el trabajo propio y el de los demás. Sin embargo, el otro grupo recibió una metodología donde no se llevaba a cabo el reparto de notas, trabajando de manera individual en gran parte del curso.

\section{Análisis empleado}

Se ha desarrollado un análisis cuantitativo en el que se realiza, por un lado, un análisis descriptivo para conocer la percepción de cada uno de los grupos sobre la evaluación recibida. Por otro lado se realiza un análisis estadístico inferencial mediante el paquete estadístico SPSS 20.0. Se han elaborado correlaciones de Pearson entre cada uno de los tres factores para ver la relación encontrada entre los mismos. También se han realizado tablas de contingencia $y \stackrel{2}{*}$, determinando el grado de significatividad que se alcanza en las respuestas relativas a la responsabilidad individual y grupal, así como la coherencia y el proceso de guiado a lo largo de las asignaturas. Por último, se han utilizado ANOVAS con el fin de observar el grado de influencia que tiene el número de veces que

Tabla 3

\begin{tabular}{|c|c|c|c|}
\hline \multirow{2}{*}{\multicolumn{4}{|c|}{ Grupos evaluación tradicional }} \\
\hline & & & \\
\hline Factor $1 /$ Factor 2 & 307 & .042 & .722 \\
\hline Factor $1 /$ Factor 3 & 307 & .418 & .321 \\
\hline Factor $2 /$ Factor 3 & 307 & .413 & .243 \\
\hline \multicolumn{4}{|c|}{ Grup os reparto de notas } \\
\hline Factor 1/Factor 2 & 335 & . .112 & . 610 \\
\hline Factor $1 /$ Factor 3 & 335 & .520 & .003 \\
\hline Factor 2/Factor 3 & 335 & . .422 & .231 \\
\hline
\end{tabular}

Tabla 4

Relación entre ítems de los tres factores vinculados con la regulación del trabajo en el proceso y la responsabilidad ind ividual y grupal ejercida para cada uno de los grupos

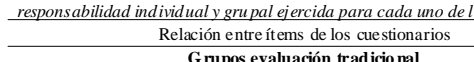

\begin{tabular}{|c|c|c|c|}
\hline & \\
\hline Grupos evaluación tradicio nal & $?^{2}$ & gl & \\
\hline Evaluación centrada en el proceso (F1)/responsabili dad ind ividual (F1) & 18.123 & 5 & .122 \\
\hline Distribución del trabajo (F2)/ resp onsabilidad grupal (F1) & 12.213 & 4 & .003 \\
\hline Coherencia con las tareas desarrollados $(F$ 2)/aprendizaje más guiado $(F 3)$ & 9.123 & 4 & .245 \\
\hline Co mprensión del sistema de evaluación (F2) dificulta & 11.535 & & 213 \\
\hline Grupos reparto de no tas & $?^{2}$ & $g l$ & \\
\hline Evaluación centrada en el proce. & 16.257 & & .002 \\
\hline Distribución del trabajo (F2)/ resp onsabilid & 13. 565 & & .015 \\
\hline Coherencia con las & 11.311 & 5 & 135 \\
\hline
\end{tabular}

el alumnado haya participado en trabajos en grupo a lo largo de su carrera para su percepción sobre el aprendizaje auténtico generado. Esta variable dependiente también se relaciona con otras independientes cómo la edad del alumnado o el curso en el que se encuentre.

\section{Resultados}

\section{Análisis descriptivos}

Se presentan los datos agrupados en cada uno de los tres factores en los que se divide el cuestionario (tabla 2)

Se observa cómo en el factor uno, relativo a la obtención de aprendizajes auténticos, los resultados son diferentes para cada uno de los grupos, existiendo una diferencia significativa en la comparación de medias. Del mismo modo, llama la atención la diferencia de más de un punto del factor tres, relativo a la regulación del proceso y continuidad, existiendo de nuevo diferencias significativas entre ambos grupos Finalmente en el factor dos apenas existen diferencias entre un grupo y otro, lo que muestra que la claridad en los sistemas de evaluación ha sido elevada para los dos grupos.

\section{Análisis Inferencial}

\section{Correlaciones}

Se observa cómo únicamente se encuentra una relación significativa entre el factor uno y tres (tabla 3 ) en el grupo de reparto de notas $\left(\mathrm{r}_{(335)=}\right.$ $.520, p<.003)$. Esto provoca una linealidad en las respuestas vinculadas a la percepción del alumnado sobre la obtención de un aprendizaje más auténtico y la necesidad de realizar un seguimiento continuo a lo largo de la asignatura, algo que no sucede en los grupos que han recibido una evaluación tradicional.

\section{Tablas de contingencia $y \chi^{2}$}

Se mide el nivel de significatividad existente en los ítems relativos a la motivación hacia el aprendizaje y la implicación y el grado de seguimiento que requiere el sistema de evaluación formativa empleado. Los ítems son extraídos de cada uno de los tres factores de análisis en el estudio (tabla 4).

Se observa cómo dentro de los grupos que han recibido una evaluación tradicional se obtiene significatividad en los ítems relacionados con la distribución del trabajo y la responsabilidad grupal $\left(\chi^{2}{ }_{(307)=} 18.123\right.$, $p=.122$ ), encontrándose ambos aspectos por debajo del valor tres «algo» en el cuestionario. Sin embargo, dentro de los grupos de reparto de notas se alcanza una significatividad en los ítems relacionados con la responsabilidad individual y grupal y la necesidad de regular el trabajo a lo largo del proceso (tanto el ítem de responsabilidad individual como el de grupal, una vez realizado el análisis de regresión múltiple, se comprueba que son los que más contribuyen al factor uno de «aprendizaje auténtico» con una $\mathrm{R}$ de .435). Además el alumnado percibe relación entre una mayor dificultad a lo largo del proceso y la necesidad de comprenderlo desde el inicio $\left(\chi_{(335)=}^{2} 11.223, p=.012\right)$.

\section{ANOVAS}

Se realizó un ANOVA de una vía para grupos independientes, con el fin de comprobar si existe diferencia estadísticamente significativa en la creación de un aprendizaje auténtico, según variables como trabajo en grupo, edad y curso. Por ello, a partir de los ítems de los tres factores que se vinculan con el aprendizaje, se ha creado una variable de escala denominada «aprendizaje auténtico recibido» con el fin de relacionarla con las siguientes variables independientes categóricas. En la primera se analiza el número de veces que el alumnado ha participado en trabajos

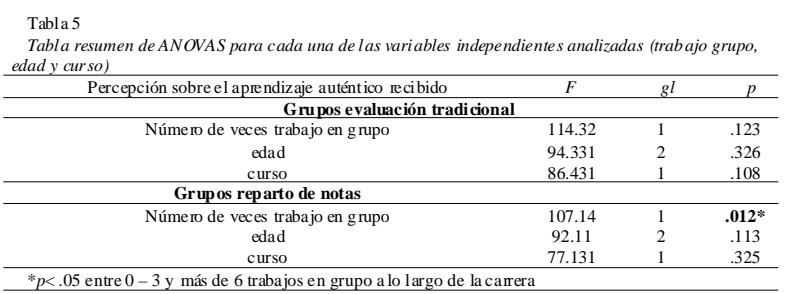


de grupo, dividiéndose en 1- «entre cero y tres veces»; 2- «entre cuatro y seis veces»; 3- «más de seis veces». La segunda es referente a la edad, quedando dividida en 1- «entre 18y 21»; 2- «22 y 25»; 3- «más de 25». Finalmente la tercera variable independiente atiende al curso en el que se imparta la asignatura, atendiendo a 1- « $1^{\circ}$ curso»; $2-« 2^{\circ}$ curso», 3- « $3^{\circ}$ curso», 4- « $4^{\circ}$ curso».

Tras realizar un análisis post hoc, se observa cómo únicamente se obtiene significatividad en la variable relativa al número de veces que se ha trabajado en grupo a lo largo de la carrera para los estudiantes que han participado en el reparto de notas $\left(\mathrm{F}_{(335)}=107.14 p<.012\right)$ (tabla 5) Esto quiere decir que el alumnado que ha participado más de seis veces de manera grupal, percibe que la técnica del reparto de notas supone un «aprendizaje auténtico», habiendo diferencia significativa con el que solamente ha participado entre cero y tres.

\section{Discusión}

Hemos observado cómo los factores extraídos en el estudio de la creación de un aprendizaje auténtico y la regulación del trabajo a lo largo del proceso son percibidos por el alumnado de manera más positiva en los grupos que han participado en el reparto de notas. Sin embargo, el factor relativo a la claridad en el sistema de evaluación se percibe por el alumnado de los dos grupos de manera similar. Delgado (2005) establece en esta línea la necesidad de que los sistemas de evaluación sean definidos claramente desde el comienzo, para así ser comprendidos por el alumnado y que le permita trabajar de manera sistemática.

Por otro lado, y dentro de los grupos que han participado en el reparto de notas, se ha obtenido una correlación significativa entre los factores relacionados con la obtención de un aprendizaje auténtico y la regulación del trabajo y continuidad a lo largo de la asignatura. Este hecho demuestra la vinculación directa entre las estrategias evaluativas que requieren de la responsabilidad del alumnado en su proceso de aprendizaje y la continuidad en las tareas. En este sentido, en los grupos que han participado en vías de evaluación tradicional no se ha obtenido ninguna correlación entre factores. Harald y Mulder (2012) comentan que el alumnado que se encuentra acostumbrado a vías de evaluación tradicionales, basadas fundamentalmente en la memorización de contenidos, no suele asociarlo con una continuidad en el proceso.

Continuando con los grupos que han recibido una evaluación tradicional, en el estudio se obtiene como relacionan significativamente los ítems vinculados a la necesidad de distribuir el trabajo a lo largo del proceso y la responsabilidad del trabajo en grupo. Ambos grados de respuesta tienen niveles bajos, lo que demuestra que en las vías tradicionales no es necesario planificar las tareas periódicamente, ya que el proceso normalmente se enfoca hacia calificaciones finales de carácter individual (Carvalho, 2012). Por el contrario, los grupos que han participado en el reparto de notas perciben la necesidad de realizar un mayor seguimiento, además de una responsabilidad individual y grupal implícita en las tareas desarrolladas. Esta es una característica que debe estar implícita en todo trabajo de grupo que se lleve a cabo, ya que si el alumnado no es capaz de delimitar roles y repartir funciones de manera comprometida, es difícil que el trabajo sea proporcionado y justo en su ejecución (Loureiro, et al., 2012). Sin embargo, es también en los grupos de reparto de notas donde se manifiesta una significatividad en las respuestas relativas a la dificultad de la comprensión del sistema y de la superación de la asignatura. En esta línea, algunas experiencias demuestran que en las vías tradicionales, en multitud de casos, el alumnado únicamente utiliza el criterio de la cantidad de materia que tiene estudiar en el examen para determinar si la asignatura es más o menos difícil (Ponn, et al., 2009).

Finalmente, se ha demostrado en la investigación cómo el alumnado que más veces ha realizado trabajos de grupo a lo largo de la carrera percibe el reparto de notas como una técnica que permite la adquisición de un «aprendizaje auténtico». En gran parte es debido a que el alumnado obtiene una serie de bagajes que son necesarios aplicar en cualquier otro ámbito de la vida real como: emitir juicios de valor objetivos a los demás, registrar el trabajo realizado semanalmente o evaluar las aportaciones de cada miembro del grupo según las tareas van teniendo más calidad (Walker, Topping \& Rodrigues, 2008). Por el contrario, la edad que tenga el alumnado o el curso en el que se encuentre la asignatura no se relaciona con ese tipo de aprendizaje. Esto se contrapone a las ideas que establecen algunos autores de que cuanta más autonomía y experiencia tenga el alumnado más valorará las nuevas técnicas de aprendizaje (Trigueros, et al., 2011).

\section{Conclusiones}

Respecto al primer objetivo, se observa cómo existen diferencias significativas entre las medias de ambos grupos sobre la percepción del aprendizaje auténtico y la implicación en el proceso de aprendizaje, ambos superiores en el grupo que participó en el reparto de notas. En relación al segundo objetivo, se observa en el estudio cómo los grupos que han participado en el reparto de notas perciben el proceso con una mayor responsabilidad, tanto individual como grupal, entendiendo que es necesario regular el trabajo desde el comienzo, algo que no sucede en la vía tradicional. Por otro lado, y dando respuesta al tercer objetivo, se comprueba cómo el hecho de haber realizado más de seis veces trabajos en grupo a lo largo de la carrera incide directamente en la percepción sobre el aprendizaje auténtico generado. Esto es algo que sucede únicamente en los grupos que han participado en el reparto de notas. Finalmente, las variables de edad y curso no influyen en la percepción de aprendizaje auténtico generado, ni en el grupo que ha recibido una evaluación tradicional ni en el que ha participado en el reparto de notas.

Sería interesante en futuros estudios analizar cuál es la percepción del profesorado ante las diferentes técnicas evaluativas empleadas, contrastándolas con las del alumnado una vez finalizada la asignatura.

\section{Referencias}

Agustí, M., Suárez-Ojeda, M., \& Baeza, M.M. (2009). El Portafolio Docente en la auto y co-evaluación del profesorado universitario: Hacia una nueva estrategia de evaluación institucional. XXI, Revista de Educación, 11, 137-154.

Argudín, Y. (2000). La Educación Superior para el siglo XXI. DINAC, 36, 16-25.

Barba, J.J. (2013). La investigación cualitativa en educación en los comienzos del siglo XXI. En A. Giráldez y M. Díaz (coords.) Investigación Cualitativa en Educación Musical (pp. 23-38). Barcelona: Graó

Barnett, R. (2001). Los límites de la competencia. El conocimiento, la educación superior y la sociedad. Barcelona: Gedisa.

Bautista, J.M., Mora, B., \& Gata, M. (2005). Los temas fundamentales del debate en torno al EEES. Recuperado de http://www.educaweb.com/ EducaNews/interface/asp/web/NoticiesMostrar.asp?NoticiaID=409\& SeccioID=639.

Badura, A., Millard, M., \& Shah, K. (2008). Are Peer Educators Really Peers? Journal of American College Health, 56, 566-568.

Boud, D., \& Falchikov, N. (2007). Rethinking Assessment in Higher Education. Learning for the long term. Oxon. Routledge.

Carvalho, C. (2012). Students' perceptions of fairness in peer assessment: evidence from a problem-based learning course. Teaching in Higher Education, 21, 212-221.

Castejón, F.J., Santos, M.L., \& Palacios, A. (2013). Cuestionario sobre metodología y evaluación en formación inicial en educación física. Revista Internacional de Medicina y Ciencias de la Actividad Física y el Deporte, 7(2), 32-55.

Castro, M., Gómez, A., \& Macazaga, A.M. (2014). Aprendizaje dialógico y grupos interactivos en educación física. Retos. Nuevas tendencias en Educación Física, Deporte y Recreación, 25, 174-179. Recuperado de file:///C:/Users/David\%20portatil/Downloads/DialnetAprendizajeDialogicoYGruposInteractivosEnEducacion4555265.pdf

Chica, E. (2011). Una propuesta de evaluación para el trabajo en grupo mediante rúbrica. Escuela Abierta, 14, 67-81.

Declaración De La Sorbona (1998). Declaración conjunta para la armonización del diseño del Sistema de Educación Superior Europeo. París, 25 de mayo de 1998. Recuperado de http:// www.informatica.uma.es/ETSIIPub/Cumbres/DeclaracionSorbona.pdf Delamare Le Deist, F., \& Winterton, J. (2005). What is competence? 
Human Resource Development International, 8(1), 27-46.

Delgado, A. (coord.) (2005). Competencias y diseño de la evaluación continua y final en el EEES. Madrid: Dirección General de Universidades. Recuperado de http://www.mec.es/univ/proyectos2005/ EA2005-0054.pdf

Dochy, F., Segers, M., \& Dierick, S. (2002). Nuevas vías de aprendizaje y enseñanza y sus consecuencias: una era de evaluación. Red Estatal de Docencia Universitaria, 2(1), 13-29.

Gil, J., \& Chiva, O. (2014). Una experiencia de aprendizaje-servicio en la asignatura «Bases anatómicas y fisiológicas del movimiento» del Área de Didáctica de la Expresión Corporal. Retos. Nuevas tendencias en Educación Física, Deporte y Recreación, 26, 122-127. Recuperado de http://www.retos.org/numero_26/122-127.pdf

González, J., \& Wagenaar, R. (2003). Tuning Educational Structures in Europe. Informe Final. Fase 1. Bilbao: Universidad de Deusto.

Hammersley, L., \& Orsmond, P. (2007). Evaluating our peers: is peerobservation a meaningful process? Studies in Higher Education, 29, 489-503.

Hamodi, C., \& López, A. T. (2012). La evaluación formativa y compartida en la Formación Inicial del Profesorado desde la perspectiva del alumnado y de los egresados. Psychology, Society, \& Education, 4, 99-112.

Harald, S., \& Mulder, A. (2012). Collaborative learning through formative peer review: pedagogy, programs and potential. Computer Science Education, 22, 343-367.

Hernanz, M. L., \& Rosselló, G. (coord.). (2003). Marc general per a la integració europea. Barcelona: Agència de Qualitat del Sistema Universitari de Catalunya.

Heywood, L., Gonczi, A., \& Hager, P. (1992). Guide to development of competence-based standards for professions. Canberra: Australian Government Publishing Service.

Jiménez, G. (2006). Obtención de notas individuales a partir de una nota de grupo mediante una evaluación cooperativa. Revista Iberoamericana de Educación, 38, 1-15.

Kerr, N. L., \& Bruun, S. E. (1983). Dispensability of member effort and group motivation losses: Free-rider effects. Journal of Personality and Social Psychology, 44, 78-94.

Liu, N. F., \& Carless, D. (2006). Peer feedback: the learning element of peer assessment. Teaching in Higher Education, 11, 279-290.

López-Pastor, V. M. (coord.) (1999). Educación Física, evaluación y reforma. Segovia: Diagonal.

López-Pastor, V. M. (2008). Implementing a Formative and Shared Assessment System in Higher Education Teaching. European Journal of Teacher Education, 31, 293-311.

López-Pastor, V. M. (2011). El papel de la evaluación formativa en la evaluación por competencias: aportaciones de la red de evaluación formativa y compartida en docencia universitaria. Revista de Docencia Universitaria, 9(1), 159-173. Recuperado de file://C:/Users/ David\%20portatil/DownloadsDialnetElPapelDeLaEvaluacion FormativaEnLaEvaluacionPorCom-4018947.pdf

López-Pastor, V.M., Castejón, J., \& Pérez-Pueyo, (2012). ¿Implicar al alumnado en la evaluación en la formación inicial del profesorado? Un estudio de caso de evaluación entre iguales de un examen. Multidisciplinary Journal of Educational Research, 2, 77-201. Recuperado de file://C:/Users/David\%20portatil/Downloads/141-8425-PB.pdf

López-Pastor, V. M., \& Gea, J.M. (2009). Innovación, discurso y racionalidad en educación física. Revisión y prospectiva. Revista Internacional de Medicina y Ciencias de la Actividad Física y el Deporte, 10(38), 245-270.

López-Pastor, V. M. \& Palacios, A. (2012). Percepción de los futuros docentes sobre los sistemas de evaluación de sus aprendizajes. Revista Teoría de la Educación: Educación y Cultura en la Sociedad de la Información, 13, 317-340

Loureiro, M., Pombo, L., \& Moreria, A. (2012). The quality of peer assessment in a wiki-based online context: a qualitative study. Educational Media International, 49, 139-144.

Maclellan, E. (2004). How convincing is alternative assessment? Assessment and Evaluation in Higher Education, 29(3), 311-321.

McMahon, T. (2010). Peer feedback in a undergraduate programme: Using action research to overcome students' reluctante to criticise. Educational Action Research, 18, 273-288.

MEC (2006). Directrices para la elaboración de Títulos Universitarios de Grado y Máster (Propuesta, de 21 de diciembre, de la Secretaría de estado de Universidades e Investigación). Madrid: MEC.
Panitz, T. (1997). Collaborative versus Cooperative Learning: Comparing the Two Definitions Helps Understand the Nature on Interactive Learning. Cooperative Learning and College Teaching, 8, 37-41.

Pérez-Pueyo, A.; Julían, J.A., \& López-Pastor, V. M. (2009). Evaluación formativa y compartida en el Espacio Europeo de Educación Superior (EEES). En V. M. López-Pastor (coord.) La Evaluación Formativa y Compartida en Docencia Universitaria: propuestas, técnicas, instrumentos y experiencias» (pp. 19-44). Madrid: Narcea.

Perrenoud, P. (2004). Diez nuevas competencias para enseñar. Barcelona: Graó.

Plaza, I., Bosque, J.M., Cerezo, P., Olmo, M., \& Viseras, C. (2012). La evaluación formativa y compartida como aspecto fundamental en la formación del profesorado novel universitario. Experiencia en las Facultades de Ciencias y Farmacia de la Universidad de Granada. Psychology, Society, \& Education, 4, 59-72.

Ponn, W., McNaught, C., Lam, P., \& Kwan, S. (2009). Improving assessment methods in university science education with negotiated self- and peer-assessment. Assessment in Education: Principles, Policy \& Practice, 3, 331-346.

Real Decreto 55/2005, de 21 de enero, por el que se establece la estructura de las enseñanzas universitarias y se regulan los estudios universitarios oficiales de Grado. Boletín Oficial del Estado, 21, de 25 de enero de 2005, 2842-2846.

Rivero, A., \& Oliván, J. (2006). Los itinerarios competenciales. En B. Learreta, (coord.), La coordinación del profesorado ante las demandas del Espacio Europeo de Educación Superior: el caso de la Facultad de Ciencias de la Actividad Física y el Deporte en la Universidad Europea de Madrid. Madrid: Universidad Europea de Madrid.

Rué, J., \& Martínez, M. (2005). Las titulaciones UAB en el Espacio Europeo de Educación Superior. Sistema Europeo de Transferencia de Créditos. Colección Eines, 1. Barcelona: Universidad Autónoma de Barcelona.

Salganik, L., Rychen, D., Moser,V., \& Konstant, J.W. (1999). Definition and Selection of Competencies: Analysis of Theoretical and Conceptual Foundations. Neuchâtel: SFSO/ OECD/ ESSI.

Sanmartí, N. (2007). (10 ideas clave) Evaluar para aprender. Barcelona: Grao.

Sheingold, K., Hawkins, J., \& Char, C. (1984). I'm the thinkist, you're the typist: The interactions of technology and the social life of classroom. Journal of Social Issues, 40(3), 49-61.

Slavin, R. (1983). Cooperative Learning. Longman: Canada.

Soler, P., \& Enrique, A.M. (2012). Reflexión sobre el rigor científico en la investigación cualitativa. Estudios sobre el mensaje periodístico, 18(2), 878-888.

Tolmie, A., Topping, K., Christie, D., Donaldson, C., Howe, C., Jessiman, E., Livingston, K., \& Thurston, A. (2010). Social effects of collaborative learning in primary schools. Learning and Instruction, 20, 177-191.

Torrance, H. (ed) (1994). Evaluating Authentic Assessment: Problems and Posibilitéis in New Approaches to Assessment. Buckingham: Open University Press.

Trigueros, C., Rivera, E., \& De la Torre, E. (2011). La evaluación en el aula universitaria: del examen tradicional a la autoevaluación. Revista Internacional de Medicina y Ciencias de la Actividad Física y el Deporte, 12(47), 473-491.

Trueba, S. (2010). Relato de una experiencia de autoevaluación y autocalificacióndel alumnado en la educación superior. Revista Ser Corporal, 4, 18-28.

Velázquez, C. (2013). Análisis de la implementación del aprendizaje cooperativo durante la escolarización obligatoria en el área de Educación Física. Tesis doctoral sin publicar. Universidad de Valladolid.

Vernetta, M., López, J., \& Delgado, M. A. (2009). La coevaluación en el aprendizaje de las habilidades gimnásticas, en el ámbito del espacio europeo universitario. Motricidad. European Journal of Human Movement, 23, 123-141.

Walker, D., Topping, K., \& Rodrigues, S. (2008). Student reflections on formative e-assessment: expectations and perceptions. Learning, Media and Technology, 33(3), 221-234.

Wimshurst, L., \& Manning, S. (2012). Feed-forward assessment, exemplars and peer marking: evidence of efficacy. Assessment \& Evaluation in Higher Education, 1, 1-15.

Zabalza, M.A. (2012). La Universidad de las competencias. REDU. Revista de Docencia Universitaria. 10(2), 11-14. 